\title{
The Influence of Boundary Conditions on Resonant Frequencies of Cavities in 3-D FDTD Algorithm Using Non-orthogonal Co-ordinates
}

\author{
Li Zhao, Lin S. Tong \\ Research Institute of Electronics, Southeast University, Nanjing 210018, P.R. China \\ Richard G. Carter \\ Engineering Department, Lancaster University, Lancaster LA1 4YR, UK
}

\begin{abstract}
The 3-dimensional finite-difference time-domain method in non-orthogonal co-ordinates (non-standard FDTD) is used to calculate the frequencies of resonators. The numerical boundary conditions of the method are presented. The influences of boundary conditions and discrete meshes on the numerical accuracy are investigated. We present the nonstandard FDTD method using the boundary-orthogonal mesh and equivalent dielectric constant so that the error is reduced from $8.6 \%$ to $3.0 \%$ for the cylindrical cavity loaded by a dielectric button.
\end{abstract}

\section{INTRODUCTION}

The resonant frequency is always an essential parameter when 3-dimensional microwave or RF cavities, such as oscillators, filters and accelerators, are designed. The accurate study of resonant frequencies has been an active area in computational electromagnetics. Many algorithms have been developed to calculate the modes of cavities [1] [3]. Over years finite-difference time-domain method on orthogonal Yee's mesh (standard FDTD) has been used successfully to work out the frequencies of resonant structures [4]-[6]. When standard FDTD method employs rectangular or cylindrical staircase mesh, the complicated boundaries of physical domain can not be fitted by the discrete mesh, especially if the boundary has small radii of curvature. In this case we have to refine the discrete mesh to ensure the numerical accuracy of FDTD method.

Recently an FDTD algorithm in non-orthogonal coordinates (non-standard FDTD) has been developed to investigate the scattering problems and resonant frequencies of dielectric-loaded cavities [7]-[11]. The regions with curved segments can be discretized well by non-orthogonal numerical cells in non-standard FDTD method. Working on fewer computing cells, the method has a potentiality of saving memory and CPU time. However, the boundary conditions of electromagnetic fields in non-orthogonal coordinate are more complicated than those in orthogonal coordinate. The simple treatment on boundary conditions may cause some extra errors. In this paper we discuss the boundary conditions of the non-standard FDTD algorithm.

Manuscript received November 1, 1993
Discrete components of electromagnetic fields are obtained on the curved electric wall and magnetic wall. According to the discussion, we introduce the boundary-orthogonal mesh and equivalent dielectric constant on the interfaces of media. Comparing our results with Harms' [11], we find that the error is reduced from $8.6 \%$ to $3.0 \%$ for the cylindrical cavity loaded by a dielectric button. The modes in resonators and filters are also calculated on boundary-orthogonal mesh (BOM) and on general non-orthogonal mesh respectively. On BOM the errors of frequencies at which the electric field is stronger near the boundary are reduced by a factor about $1 \%$. Our results show good agreements with the theoretical and numerical results.

\section{FDTD METHOD IN NON-ORTHOGONAL CO-ORDINATES AND ITS NUMERICAL BOUNDARY COOOOOONDITIONS}

In this section the non-standard FDTD method is briefly described, and then the boundary conditions are investigated on the electric wall, magnetic wall and interface between dielectric regions. The numerical components of electromagnetic fields are given on the boundaries to satisfy the implement of finite difference method.

\section{A. The Brief Description of Non-Standard FDTD Method}

In the isotropic and lossless media Maxwell's curl equations with source-free can be written as

$$
\begin{aligned}
& \nabla \times \mathbf{H}=\partial \mathbf{D} / \partial t, \\
& \nabla \times \mathbf{E}=-\partial \mathbf{B} / \partial t,
\end{aligned}
$$

where $\mathbf{D}=\varepsilon \mathbf{E}, \mathbf{B}=\mu \mathbf{H}$. Equation (1) and (2) can be discretized by the central difference in a non-orthogonal discrete cell, shown in Fig. 1, in accordance with the curvilinear coordinate theory. We define the covariant components of the electric field $\mathbf{E}$ as the tangential components at the centre of the cell's edges, and define the contravariant components of the magnetic field $\mathbf{H}$ as the normal components at the centre of the cell's surfaces (see Fig. 1). Although the discrete cells are irregular and non-orthogonal, we can numerically transform them into the cubic cells with the unit increment so that the implement of non-standard FDTD method becomes easy. Then the time domain $[0, T]$ is divided into $\mathrm{N}$ 


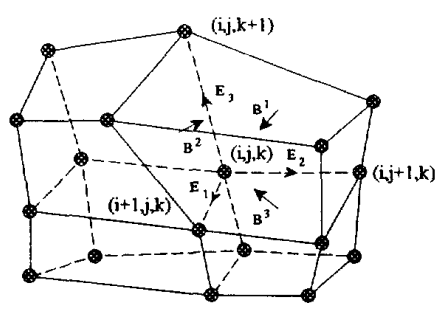

Fig. 1. The non-orthogonal discrete cell.

intervals, i.e. $\Delta t=T / N$. If we denote any function of discrete space and time as

$$
F\left(i \Delta u^{1}, j \Delta u^{2}, k \Delta u^{3}, n \Delta t\right)=F_{i, j, k}^{(n)},
$$

the component forms of non-standard FDTD method can be formulated, such as

$$
\begin{aligned}
& E_{i+\frac{1}{2}, j, k}^{l(n+1)}=E_{i+\frac{1}{2}, j, k}^{1(n)}+(\Delta t / \varepsilon)\left(\sqrt{g_{11} / g}\right)_{i+\frac{1}{2}, j, k} \\
& \cdot\left(h_{3, i+\frac{1}{2}, j+\frac{1}{2}, k}-h_{3, i+\frac{1}{2}, j-\frac{1}{2}, k}-h_{2, i+\frac{1}{2}, j, k+\frac{1}{2}}+h_{2, i+\frac{1}{2}, j, k-\frac{1}{2}}\right)^{\left(n+\frac{1}{2}\right)}, \\
& H_{i, j+\frac{1}{2}, k+\frac{1}{2}}^{1\left(n+\frac{1}{2}\right)}=H_{i, j+\frac{1}{2}, k+\frac{1}{2}}^{1\left(n-\frac{1}{2}\right)}-(\Delta t / \mu)\left(\sqrt{g_{11} / g}\right)_{i, j+\frac{1}{2}, k+\frac{1}{2}} \\
& \cdot\left(e_{3, i, j+1, k+\frac{1}{2}}-e_{3, i, j, k+\frac{1}{2}}-e_{2, i, j+\frac{1}{2}, k+1}+e_{2, i, j+\frac{1}{2}, k}\right)^{(n)}
\end{aligned}
$$

where $\mathrm{i}, \mathrm{j}, \mathrm{k}$ are integers, $\Delta \mathrm{u}^{1}, \Delta \mathrm{u}^{2}, \Delta \mathrm{u}^{3}$ are the increments of the curvilinear coordinates and equal to $1, \mathrm{E}^{1}, \mathrm{H}^{1}$ are the contravariant components of the electromagnetic vectors, $\mathrm{e}_{\mathrm{m}}, \mathrm{h}_{\mathrm{m}}(\mathrm{m}=1,2,3)$ are the covariant components of the electromagnetic vectors, $V_{g}$ is Jacobian transformation, the component $\mathrm{g}_{\mathrm{ij}}$ of the tensor matrix is given by dot product of covariant base vector $a_{i}$ and $a_{j}$, i.e. $g_{i j}=a_{i} \cdot a_{j}$. The other components can be obtained by permutation of indices.

It is found from (4) and (5) that the covariant components must be transformed into contravariant components to form self-consistent iterative procedure. By means of the vector operation the covariant component of a vector $\mathbf{F}$ is written as

$$
f_{i}=\sum_{j=1,3}\left(g_{i j} / \sqrt{g_{j j}}\right) F^{j} \quad(\mathrm{i}=1,2,3),
$$

where $\mathrm{F}^{\mathrm{j}}$ is the normalized contravariant component. One covariant component is related to three contravariant components in (6). However only one component of fields is set up at the discrete positions in Fig. 1. We have to use the interpolation to obtain unknown components, for example,

$$
\begin{array}{r}
e_{1}=\sqrt{g_{11}} E^{1}+ \\
\frac{g_{12}}{4 \sqrt{g_{22}}}\left(E_{i+1, j+\frac{1}{2}, k}^{2}+E_{i, j+\frac{1}{2}, k}^{2}+E_{i+1, j-\frac{1}{2}, k}^{2}+E_{i, j-\frac{1}{2}, k}^{2}\right) \\
\frac{g_{13}}{4 \sqrt{g_{33}}}\left(E_{i+1, j, k+\frac{1}{2}}^{3}+E_{i, j, k+\frac{1}{2}}^{3}+E_{i+1, j, k-\frac{1}{2}}^{3}+E_{i, j, k-\frac{1}{2}}^{3}\right)
\end{array}
$$$$
\text { at the point }(i+1 / 2, j, k) \text {. }
$$

The similar procedure is applied to give the covariant components of magnetic field. Permuting indices and substituting all covariant components into (4) and (5), we can formulate the iterative equations of non-standard FDTD method, in which the critical time step is as follows [10]

$$
\Delta t \leq \max \left(c \sqrt{\sum_{i} \sum_{j}\left(g_{j m} g_{k n}-g_{j n} g_{k m}\right)}\right),
$$

where $i, 1=1,2,3,(i, j, k),(1, m, n)$ cycle.

\section{B. The Numerical Boundary Conditions of Non-Standard FDTD Method}

In standard FDTD algorithm the tangential and normal components of electromagnetic fields can easily satisfy the boundary conditions of a closed domain, even when the magnetic wall coincides with the discrete cells. However, in non-standard FDTD method the boundary conditions become complicated because of the non-orthogonality of the discrete mesh. If the surface $s_{i}$ on which coordinate $u^{i}$ is constant, shown in Fig. 2, is an electric wall, the component $B^{i}$ is equal to zero on the $s_{i}$ for $B^{i}$ is a normal component, and the tangential electric components can be written as

$$
e_{j}=0, e_{k}=0 \text {. }
$$

Using (6) we get equations about contravariant components of electric field $\mathbf{E}$ on the surface $s_{\mathbf{i}}$

$$
\begin{aligned}
& \sqrt{g_{i j}} E^{j}+\left(g_{j k} / \sqrt{g_{k k}}\right) E^{k}+\left(g_{j i} / \sqrt{g_{i i}}\right) E^{i}=0, \\
& \left(g_{k j} / \sqrt{g_{i j}}\right) E^{j}+\sqrt{g_{k k}} E^{k}+\left(g_{k i} / \sqrt{g_{i i}}\right) E^{i}=0 .
\end{aligned}
$$

To eliminate the extra degree of freedom in (10) we introduce BOM into the non-standard FDTD method so that the coordinate line $u^{i}$ is normal to the surface $s_{i}$. Thus $g_{j i}$ and $\mathrm{g}_{\mathrm{ki}}$ will disappear. Investigating the determination of the coefficient matrix of equation (10)

$$
\operatorname{det}|G|=\left(g_{j j} g_{k k}-g_{j k}^{2}\right) / \sqrt{g_{j j} g_{k k}},
$$

we find that $\operatorname{det}|\mathrm{G}|$ is always greater than zero because the differential surface element $d s_{i}$ is

$$
d s_{i}=\sqrt{g_{j j} g_{k k}-g_{j k}^{2}} d u^{j} d u^{k}
$$

Therefore, (11) has zero solutions, i.e.

$$
E^{j}=0, E^{k}=0
$$

The contravariant component $\mathrm{E}^{\mathrm{j}}, \mathrm{E}^{\mathrm{k}}$ completely satisfy the boundary conditions on the electric wall. BOM and the general mesh are shown in Fig. 3.

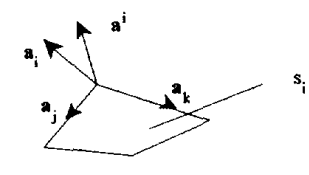

Fig. 2. The boundary surface $s_{i}$. 


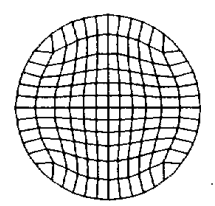

Fig. 3. (a) The boundary-orthogonal mesh.

(b) The general mesh.

Similarly, if the surface $s_{i}$ is a magnetic wall and coincides with the discrete cells we have equations about the contravariant components of magnetic field on the $s_{i}$, i.e.

$$
\begin{gathered}
\sqrt{g_{j j}} H^{j}+\left(g_{j k} / \sqrt{g_{k k}}\right) H^{k}+\left(g_{j i} / \sqrt{g_{i i}}\right) H^{i}=0, \\
\left(g_{k j} / \sqrt{g_{j j}}\right) H^{j}+\sqrt{g_{k k}} H^{k}+\left(g_{k i} / \sqrt{g_{i i}}\right) H^{i}=0 .
\end{gathered}
$$

When BOM is used, $\mathrm{H}^{\mathbf{j}}$ and $\mathrm{H}^{\mathbf{k}}$ are equal to zero on the $\mathbf{s}_{\mathbf{i}}$. However, the positions of $\mathrm{H}^{\mathrm{j}}$ and $\mathrm{H}^{\mathrm{k}}$ in the discrete cells are shifted half-cell from the magnetic wall (see Fig. 1). We use the interpolation to eliminate the components that exist in the iterative formulas and are beyond the domain, and have

$$
H_{u^{j}+u^{i} / 2}^{j}=-H_{u^{i}-u^{i} / 2}^{j}, H_{u^{i}+u^{i} / 2}^{k}=-H_{u^{i}-u^{i} / 2}^{k} .
$$

When non-standard FDTD method works on the general mesh, numerical errors that depend on the $\mathrm{E}^{\mathrm{i}}$ component and its coefficient in (10) or the $\mathrm{H}^{\mathrm{i}}$ component and its coefficient in (14), may be introduced. The use of BOM will make the boundary conditions be satisfied completely.

On the interfaces between cells the equivalent dielectric constants consist of those which are different each other in four adjacent cells (Fig. 1). Here we present an area-weighted technique to obtain better approximation to the dielectric constant. The equivalent dielectric constant at the point $(\mathrm{i}+1 / 2, \mathrm{j}, \mathrm{k})$ can be written as

$$
\varepsilon_{i+\frac{1}{2}, j, k}=\left(\Delta_{i, j, k}+\Delta_{i, j-1, k}+\Delta_{i, j-1, k-1}+\Delta_{i, j, k-1}\right) /\left(4 A_{i, j, k}\right)
$$

where $\Delta_{i, j, k}=\varepsilon_{i, j, k} s_{i+\frac{1}{2}, j+\frac{1}{2}, k+\frac{1}{2}}$,

$A_{i, j, k}=s_{i+\frac{1}{2}, j+\frac{1}{2}, k+\frac{1}{2}}+s_{i+\frac{1}{2}, j-\frac{1}{2}, k+\frac{1}{2}}+s_{i+\frac{1}{2}, j-\frac{1}{2}, k-\frac{1}{2}}+s_{i+\frac{1}{2}, j+\frac{1}{2}, k-\frac{1}{2}}$, where the area $s$ can be worked out by the integration over the discrete cells, for example,

$$
s_{i+\frac{1}{2}, j+\frac{1}{2}, k+\frac{1}{2}}=\left(\sqrt{g_{22} g_{33}-g_{23}^{2}}\right)_{i+\frac{1}{2}, j+\frac{1}{2}, k+\frac{1}{2}} .
$$

So far we have investigated the boundary conditions of electromagnetic fields in a closed domain as well as the equivalent dielectric constant on the interface between cells.

\section{NUMERICAL RESULTS AND DISCUSSION}

The time-domain response of EM fields in FDTD method should be transformed into frequency-domain results by Discrete Fourier Transformation (DFT) to obtain the resonant frequencies. The spectrum can be extracted by

$$
F_{k}(k \Delta f)=\Delta t \sum_{i=0}^{N-1} f_{i}(i \Delta t) \exp (-j 2 \pi i k / N) .
$$

The running procedure of FDTD method is as follows

(a) Set the components of electric field to zero in the domain, except on some grids, at $t=0$,

(b) Calculate the magnetic field $B$ at $t=n+1 / 2$,

(c) Calculate the electric field $\mathbf{E}$ at $\mathbf{t}=\mathbf{n}+1$, and sample $\mathbf{E}$,

(d) Repeat (b), (c) up to the required iterative times,

(e) Extract the resonant spectrum through DFT.

The BOM can be generated by equation $\nabla^{2} r=0$ [12]. There are no limitations on the shape of computing domain if the domain can be divided into arbitrary hexahedrons.

\section{A. Cylindrical Cavity}

At first, the resonant frequencies of cylindrical cavity (radius $=10 \mathrm{~cm}$, length $=10 \mathrm{~cm}$ ) are calculated on the general mesh and BOM respectively (Fig. 3). The discrete cells are $12 \times 12 \times 8$. We have the iterative times $N=2^{14}$, frequency definition $\Delta \mathrm{f}=0.009 \mathrm{GHz}$, time step $\Delta \mathrm{t}=1.37 \times 10^{-11} \mathrm{~s}$ on the general mesh. Using $B O M$, we have $\mathrm{N}=2^{13}, \Delta \mathrm{f}=0.011 \mathrm{GHz}$, $\Delta \mathrm{t}=2.18 \times 10^{-11} \mathrm{~s}$. The results are shown in Table I. The DFT spectrum is shown in Fig. 4. Comparing the results we find that the boundary orthogonality of mesh has an effect on the numerical accuracy when there is stronger electric field near the boundary. In this example the error is reduced from $1.9 \%$ to $.5 \%$ for $\mathrm{TM}_{011}$ mode when BOM is used. The numerical errors are less than $1.5 \%$ for the lowest five modes.

TABLE I

THE RESONANT FREQUENCIES OF THE CYLINDRICAL CAVITY

\begin{tabular}{cccccc}
\hline & Theory & \multicolumn{2}{c}{ General Mesh } & \multicolumn{2}{c}{ BOM } \\
\hline Mode & $\mathrm{f}(\mathrm{GHz})$ & $\mathrm{f}(\mathrm{GHz})$ & error $(\%)$ & $\mathrm{f}(\mathrm{GHz})$ & error (\%) \\
TM010 & 1.15 & 1.14 & 0.88 & 1.14 & 0.88 \\
TE111 & 1.72 & 1.74 & 1.16 & 1.70 & -1.16 \\
TM110 & 1.82 & 1.80 & 1.11 & 1.80 & 1.11 \\
TM011 & 1.87 & 1.90 & 1.60 & 1.88 & 0.05 \\
TE211 & 2.06 & 2.10 & 1.90 & 2.09 & 1.46 \\
\hline
\end{tabular}

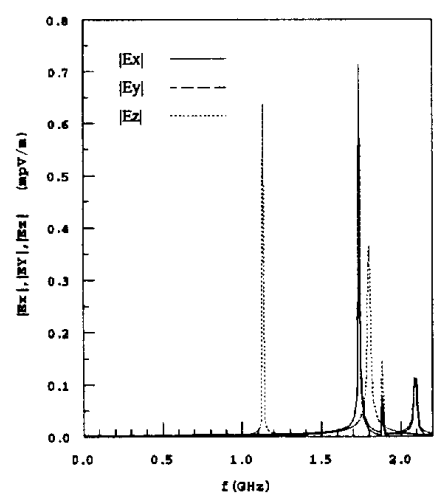

Fig. 4. The lowest resonant frequencies of the cylindrical cavity. 


\section{B. Cylindrical Cavity Loaded by Dielectric Button}

Secondly we calculate the resonant frequencies of the cylindrical cavity loaded by a dielectric button (Fig. 5) on the general mesh and BOM respectively. The discrete cells are $14 \times 14 \times 12$, shown in Fig. 6 . In the case of the general mesh we have the frequency definition $\Delta f=0.09 \mathrm{GHz}$, iterative times $\mathrm{N}=2^{14}$, time step $\Delta t=1.31 \times 10^{-12} \mathrm{~s}$. When use BOM, we have $\Delta \mathrm{f}=0.08 \mathrm{GHz}, \mathrm{N}=2^{14}, \Delta \mathrm{t}=1.59 \times 10^{-12} \mathrm{~s}$. The results are shown in Table II, and compared with the values given by mode-matching method (MM) in reference [11]. The DFT spectrum is shown in Fig. 7. Comparing our results with Harms' data that are also obtained by non-standard FDTD, we find that the error is reduced from $8.6 \%$ to $3.0 \%$ due to the introduction of BOM and equivalent dielectric constant. The errors are reduced by a factor about $1 \%$ for $\mathrm{HEll}$ and HE 12 mode that have stronger electric field near the metal boundary after BOM is made.

\section{TABLE II}

THE RESONANT FREQUENCIES OF THE CYLINDRICAL CAVITY LOADED BY A DIELECTRIC BUTTON ( $\mathrm{a}=0.8636 \mathrm{~cm}, \mathrm{~b}=1.295 \mathrm{~cm}, \mathrm{H}=0.762, \mathrm{LI}=\mathrm{L} 2=0.381 \mathrm{~cm}$, $\mathrm{e}=35.74)$

\begin{tabular}{ccccccc}
\hline & $\begin{array}{c}\text { MM } \\
{[11]}\end{array}$ & $\begin{array}{c}\text { FEM } \\
{[5]}\end{array}$ & $\begin{array}{c}\text { FDTD } \\
{[11]}\end{array}$ & $\begin{array}{c}\text { FDTD } \\
\text { general mesh }\end{array}$ & $\begin{array}{c}\text { FDTD } \\
\text { BOM }\end{array}$ & error \\
\hline Mode & $(\mathrm{GHz})$ & $(\mathrm{GHz})$ & $(\mathrm{GHz})$ & $(\mathrm{GHz})$ & $(\mathrm{GHz})$ & $(\%)$ \\
TE01 & 3.44 & 3.51 & 3.53 & 3.44 & 3.46 & 0.6 \\
HE11 & 4.27 & 4.27 & 3.90 & 4.18 & 4.23 & 0.9 \\
HE12 & 4.37 & 4.36 & 4.17 & 4.27 & 4.32 & 1.1 \\
TM01 & 4.60 & 4.54 & 4.53 & 4.46 & 4.46 & 3.0 \\
\hline
\end{tabular}

\section{CONCLUSION}

The boundary conditions of the non-standard FDTD method are presented. The boundary-orthogonal mesh and the equivalent dielectric constant are introduced. Compared with the data from other sources, the error is reduced from $8.6 \%$ to $3.0 \%$ for the cylindrical cavity loaded by a dielectric button. The frequencies of resonators and filters are also calculated on boundary-orthogonal mesh and on general mesh. In our examples the error can be reduced by a factor about $1 \%$ for those modes that have stronger electric field near the metal boundary after the boundary-orthogonal mesh is made. The results show excellent agreements with theoretical values and numerical results.

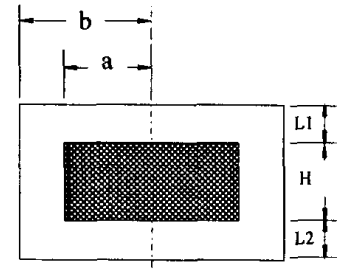

Fig. 5. The cylindrical cavity loaded by a dielectric button.

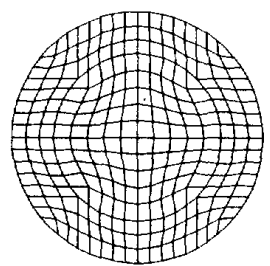

Fig. 6. The discrete mesh for Fig. 5.

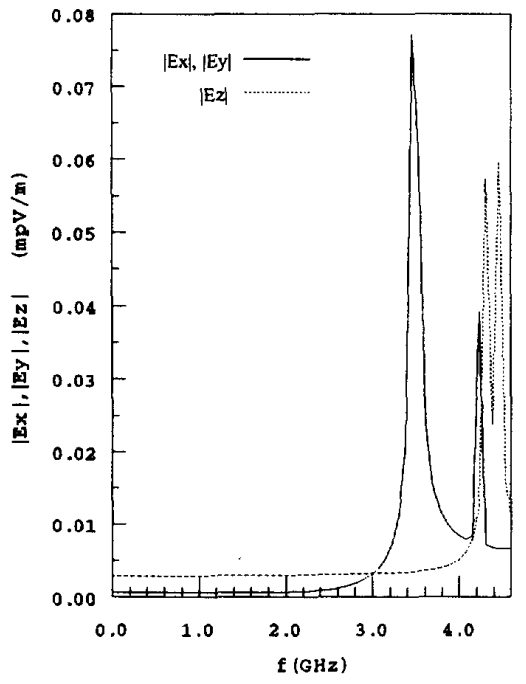

Fig. 7. The lowest resonant frequencies of cylindrical cavity loaded by a dielectric button.

\section{REFERENCES}

[1] T. Weiland, " On the numerical solution of Maxwell's equations and applications in the field of accelerator physics," Particle Accelerators, vol. 15, pp. 245-292, 1984.

[2] T. Weiland, "Three dimensional resonator mode computation by finite difference method," IEEE Trans. Magn., vol. MAG-21, pp. 2340-2343, Nov. 1985

[3] M.M. Taheri and D. Mirshekar-Syahkal, " Accurate determination of modes in dielectric-loaded cylindrical cavities using a onedimensional finite element method," IEEE Trans. Microwave Theory Tech., vol. MTT-37, pp. 1536-1541, Oct. 1989.

[4] K.S. Yee, " Numerical solutions of initial boundary value problems involving Maxwell's equations in isotropic media," IEEE Trans. Antennas Propagat., vol. AP-14, pp. 302-307, May 1966.

[5] D.H. Choi and W.J.R. Hoefer," The finite-difference time-domain method and its application to eigenvalue problems," IEEE Trans. Microwave Theory Tech., vol. MTT-34, pp. 1464-1470, Dec. 1986.

[6] A. Navarro, M.J. Nunez and E. Martin, "Study of $\mathrm{TE}_{0}$ and $\mathrm{TM}_{0}$ modes in dielectric resonators by a finite-difference time-domain method coupled with the discrete Fourier transform," IEEE Trans. Microwave Theory Tech., vol. MTT-39, pp. 14-17, Jan. 1991.

[7] R. Holland, "Finite-difference solution of Maxwell's equations in generalized nonorthogonal coordinates," IEEE Trans. Nucl. Sci., vol. NS-30, no. 6, pp. 4589-4591, Dec. 1983.

[8] M. Fusco, "FDTD algorithm in curvilinear coordinates," IEEE Trans. Antennas Propagat., vol. AP-38, pp. pp. 78-89, Jan. 1990.

[9] M.A. Fusco, M.V. Smith and L.W. Gordon, " A three-dimensional FDTD algorithm in curvilinear coordinates," IEEE Trans. Antennas Propagat, vol. AP-39, pp. 1463-1471, Oct. 1991.

[10] J.F. Lee, R. Palandech and R. Mittra, " Modeling three-dimensional discontinuities in waveguides using nonorthogonal FDTD algorithm," IEEE Trans. Microwave Theory Tech., vol. MTT-40, pp. 346-352, Feb. 1992.

[11] P.H. Harms, J.F. Lee and R. Mittra, " A study of the nonorthogonal FDTD method versus the conventional FDTD technique for computing resonant frequencies of cylindrical cavities," IEEE Trans. Microwave Theory Tech., vol. MTT-40, pp. 741-746, Apr. 1992.

[12] J.F. Thompson, Z.U.A. Warsi and C.W. Mastin, Numerical Grid Generation: Foundations and Applications, North-Holland, Amsterdam, 1985. 